\title{
Total dominating functions of graphs: antiregularity versus regularity
}

\author{
Maria Talanda-Fisher, Ping Zhang* \\ Department of Mathematics, Western Michigan University, Kalamazoo, MI 49008, USA
}

(Received: 6 November 2020. Received in revised form: 28 December 2020. Accepted: 29 December 2020. Published online: 31 December 2020.)

(C) 2020 the authors. This is an open access article under the CC BY (International 4.0) license (www.creativecommons.org/licenses/by/4.0/).

\begin{abstract}
A set $S$ of vertices in a nontrivial connected graph $G$ is a total dominating set if every vertex of $G$ is adjacent to some vertex of $S$. The minimum cardinality of a total dominating set for $G$ is the total domination number of $G$. A function $h: V(G) \rightarrow\{0,1\}$ is a total dominating function of a graph $G$ if $\sigma_{h}(v)=\sum_{u \in N(v)} h(u) \geq 1$ for every vertex $v$ of $G$. A total dominating function $h$ of a nontrivial graph $G$ is irregular if $\sigma_{h}(u) \neq \sigma_{h}(v)$ for every two vertices $u$ and $v$ of $G$. While no graph possesses an irregular total dominating function, a graph $G$ has an antiregular total dominating function $h$ if there are exactly two vertices $u$ and $v$ of $G$ such that $\sigma_{h}(u)=\sigma_{h}(v)$. It is shown that for every integer $n \geq 3$, there are exactly two non-isomorphic graphs of order $n$ having an antiregular total dominating function. If $h$ is a total dominating function of a graph $G$ such that $\sigma_{h}(v)$ is the same constant $k$ for every vertex $v$ of $G$, then $h$ is a $k$-regular total dominating function of $G$. We present some results dealing with properties of regular total dominating functions of graphs. In particular, regular total dominating functions of trees are investigated. The only possible regular total dominating functions for a nontrivial tree are 1-regular total dominating functions. We characterize those trees having a 1-regular total dominating function. We also investigate $k$-regular total dominating functions of several well-known classes of regular graphs for various values of $k$.
\end{abstract}

Keywords: domination; total domination; total dominating function; irregular, antiregular, and regular total dominating functions.

2020 Mathematics Subject Classification: 05C05, 05C69, $05 \mathrm{C} 75$.

\section{Introduction}

Domination in graphs has become one of the most popular areas of graph theory, no doubt due to its many fascinating problems and applications to modern society, as well as the sheer mathematical beauty of the subject. While this area evidently began with work by the French mathematician Claude Berge [3] in 1958 and the Norwegian-American mathematician Oystein Ore [15] in 1962, domination did not become an active area of research until 1977 with the appearance of the survey paper by Ernest Cockayne and Stephen Hedetniemi [7]. Since then a large number of variations of domination have surfaced and provided numerous applications to different areas of science and real-life problems (see [5, 12, 13]). In 1987, Hedetniemi introduced the concept of dominating functions which (a) provided an analytic method of studying this discrete concept, (b) built a connection between domination and graph labelings and colorings, and (c) gave rise to new dominating function parameters (see [4,11]). In 2019, Gary Chartrand introduced several variations of dominating functions in connection with some of the best-known concepts in graphs, including irregularity, regularity and chromaticity in graphs (see [1,2,5, 8-10]).

A vertex $u$ is said to dominate a vertex $v$ in a graph if either $u=v$ or $v$ is a neighbor of $u$. One of the many variations of domination is total domination, introduced by Cockayne, Dawes, and Hedetniemi [6]. In this variation, a vertex $u$ dominates a vertex $v$ only if $v$ is a neighbor of $u$, that is, a vertex does not dominate itself.

A set $S$ of vertices in a graph $G$ is a total dominating set for $G$ if every vertex of $G$ is adjacent to some vertex of $S$. In particular, every vertex of $S$ must be adjacent to another vertex of $S$. Therefore, a graph $G$ has a total dominating set if and only if $G$ contains no isolated vertices. Furthermore, if $S$ is a total dominating set of $G$, then the subgraph $G[S]$ induced by $S$ contains no isolated vertices. The minimum cardinality of a total dominating set for $G$ is the total domination number $\gamma_{t}(G)$ of $G$.

There is another way to look at total domination and the total domination number of a graph $G$ without isolated vertices. Let $h: V(G) \rightarrow\{0,1\}$ be a function. Then $h$ gives rise to another function $\sigma_{h}: V(G) \rightarrow \mathbb{N} \cup\{0\}$ defined by

$$
\sigma_{h}(v)=\sum_{u \in N(v)} h(u)
$$

*Corresponding author (ping.zhang@wmich.edu) 
where $\mathbb{N}$ is the set of positive integers and $N(v)$ is the neighborhood of $v$. Thus, $0 \leq \sigma_{h}(v) \leq \operatorname{deg} v \leq \Delta(G)$ for every vertex $v$ of $G$. If $\sigma_{h}(v) \geq 1$ for every vertex $v$ of $G$, then $h$ is called a total dominating function of $G$. If $h$ is a total dominating function of $G$, then the set $\mathcal{I}_{h}=\{v \in V(G): h(v)=1\}$ is a total dominating set of $G$. On the other hand, if $S$ is a total dominating set in $G$, then the function $h$ that assigns 1 to each vertex of $S$ and 0 to each vertex in $\bar{S}=V(G)-S$ is a total dominating function of $G$ with $\mathcal{I}_{h}=S$. This is illustrated in Figure 1 where a total dominating function of a graph $G$ is obtained from a total dominating set in $G$.

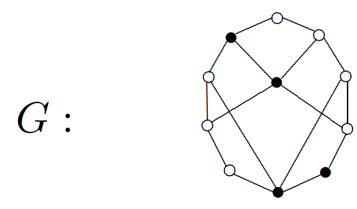

a total dominating set

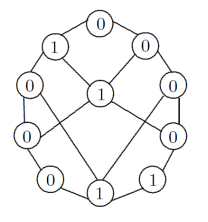

a total domintion function

Figure 1: A total dominating function in a graph.

Observation 1.1. Let $G$ be a nontrivial connected graph and let $h: V(G) \rightarrow\{0,1\}$ be a total dominating function of $G$. Then

$$
\sum_{v \in V(G)} \sigma_{h}(v)=\sum_{v \in V(G)} \operatorname{deg} v \cdot h(v)=\sum_{x \in \mathcal{I}_{h}} \operatorname{deg} x .
$$

\section{Irregular and antiregular total dominating functions}

Let $G$ be a connected graph with no isolated vertices. If $h$ is a total dominating function of $G$ such that $\sigma_{h}(u) \neq \sigma_{h}(v)$ for every two vertices $u$ and $v$ of $G$, then $h$ is called an irregular total dominating function of $G$. However, no graph possesses an irregular total dominating function.

Observation 2.1. No nontrivial connected graph possesses an irregular total dominating function.

A nontrivial graph $G$ is antiregular if exactly two vertices of $G$ have the same degree. Furthermore, for every integer $n \geq 2$, there are exactly two non-isomorphic antiregular graphs of order $n$, one of which is connected (see [1,2]). We denote the connected antiregular graph of order $n$ by $G_{n}$ and its complement by $\bar{G}_{n}$. Since the disconnected antiregular graph $\bar{G}_{n}$ of order $n \geq 2$ contains an isolated vertex, it follows that $\bar{G}_{n}$ does not have a total dominating function.

A total dominating function $h: V(G) \rightarrow\{0,1\}$ of a nontrivial connected graph $G$ of order $n$ is called antiregular if $\left|\left\{\sigma_{h}(v): v \in V(G)\right\}\right|=n-1$, that is, if there are exactly two vertices $x$ and $y$ of $G$ such that $\sigma_{h}(x)=\sigma_{h}(y)$. Consequently, if $h$ is an antiregular total dominating function of $G$, then $\left\{\sigma_{h}(v): v \in V(G)\right\}=[n-1]=\{1,2, \ldots, n-1\}$. Every connected antiregular graph has an antiregular total dominating function.

Proposition 2.1. Every connected antiregular graph has an antiregular total dominating function.

Proof. For the unique connected antiregular graph $G_{n}$ of order $n \geq 2$, define the total dominating function $f: V\left(G_{n}\right) \rightarrow$ $\{0,1\}$ by $f(v)=1$ for every vertex $v$ of $G_{n}$. Thus, $\sigma_{f}(v)=\operatorname{deg} v \geq 1$ for each $v \in V\left(G_{n}\right)$. Since $G_{n}$ is antiregular, $f$ is an antiregular total dominating function of $G_{n}$.

There are also non-antiregular graphs having an antiregular total dominating function. For example, the three graphs of Figure 2 are not antiregular but have an antiregular total dominating function, also shown in Figure 2.
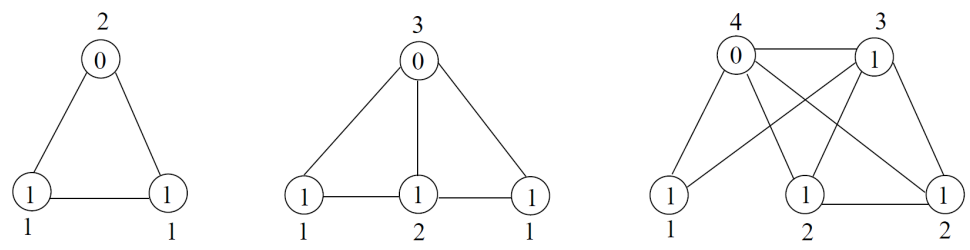

Figure 2: Non-antiregular graphs with an antiregular total dominating function.

These examples give rise to the following question.

Which non-antiregular graphs of order 3 or more have an antiregular total dominating function? 
Each of the three graphs of order $n \in\{3,4,5\}$ in Figure 2 has maximum degree $n-1$ and minimum degree 2. As we show next, this is true for all non-antiregular graphs of order 3 or more having an antiregular total dominating function.

Proposition 2.2. If a non-antiregular graph $G$ of order $n \geq 3$ has an antiregular total dominating function, then $\Delta(G)=$ $n-1$ and $\delta(G)=2$.

Proof. Let $G$ be a non-antiregular graph of order $n \geq 3$ and let $h: V(G) \rightarrow\{0,1\}$ be an antiregular total dominating function of $G$. Then $S_{h}=\left\{\sigma_{h}(x): x \in V(G)\right\}=[n-1]$. Assume, to the contrary, that either $\Delta(G) \leq n-2$ or $\delta(G) \neq 2$. First, suppose that $\Delta(G) \leq n-2$. Since $1 \leq \sigma_{h}(v) \leq \Delta(G) \leq n-2$ for every vertex $v$ of $G$, it follows that $S_{h} \subseteq[n-2]$, which is a contradiction. Consequently, $\Delta(G)=n-1$.

Next, suppose that $\delta(G) \neq 2$. We consider two cases, according to whether $\delta(G)=1$ or $\delta(G) \geq 3$.

Case 1. $\delta(G)=1$. Let $u$ be an end-vertex of $G$ and let $v \in V(G)$ such that $\sigma_{h}(v)=n-1$. Then $\operatorname{deg} v=n-1$ and $h(x)=1$ for each $x \in N(v)=V(G)-\{v\}$. On the other hand, the end-vertex $u$ is adjacent only to $v$ and so $h(v)=1$. Therefore, $h(x)=1$ for every vertex $x$ of $G$ and so $\sigma_{h}(x)=\operatorname{deg} x$ for every vertex $x$ of $G$. However then, $\{\operatorname{deg} x: x \in V(G)\}=S_{h}=[n-1]$. This implies that $G$ is an antiregular graph, which is a contradiction.

Case 2. $\delta(G) \geq 3$. Again, let $v \in V(G)$ such that $\sigma_{h}(v)=n-1$. Then $\operatorname{deg} v=n-1$ and $h(x)=1$ for each $x \in N(v)=$ $V(G)-\{v\}$. Since $\operatorname{deg} x \geq 3$ for each vertex $x$ of $G$ and $h(x) \in\{0,1\}$, it follows that $\sigma_{h}(x) \geq 2$ for every vertex $x$ of $G$. Consequently, $S_{h} \subseteq\{2,3, \ldots, n-1\}$, which is a contradiction.

Therefore, $\Delta(G)=n-1$ and $\delta(G)=2$, as claimed.

By Proposition 2.2, if $G$ is a non-antiregular graph of order $n \geq 3$ such that $\Delta(G) \leq n-2$ or $\delta(G) \neq 2$, then $G$ does not have an antiregular total dominating function. We next show that for every integer $n \geq 3$, there are exactly two graphs of order $n$ possessing an antiregular total dominating function, one of which is the connected antiregular graph $G_{n}$ of order $n$. In order to state and verify this result, we first introduce an additional definition. The join $G=F \vee H$ of two vertex-disjoint graphs $F$ and $H$ has vertex set $V(G)=V(F) \cup V(H)$ and edge set $E(G)=E(F) \cup E(H \cup\{u v: u \in V(F), v \in V(H)\}$. In particular, the graph $F \vee K_{1}$ is constructed by adding a new vertex to $F$ and joining this vertex to every vertex of $F$.

Theorem 2.1. For each integer $n \geq 3$, there are exactly two graphs of order $n$ with an antiregular total dominating function, one of which is $G_{n}$ and the other is $G_{n-1} \vee K_{1}$.

Proof. It is readily seen that $G_{3}=P_{3}$ and $K_{3}=G_{2} \vee K_{1}=P_{2} \vee K_{1}$ (shown in Figure 2) are the only graphs of order 3 having an antiregular total dominating function. Let $H$ be a graph of order $n \geq 4$ having an antiregular total dominating function $h$. Then $S_{h}=\left\{\sigma_{h}(v): v \in V(H)\right\}=[n-1]$. Let $u \in V(H)$ such that $\sigma_{h}(u)=n-1$. Then $\operatorname{deg} u=n-1$ and $h(x)=1$ for each $x \in N(u)=V(H)-\{u\}$. Since $h(u) \in\{0,1\}$, there are two possibilities.

Case 1. $h(u)=1$. Therefore, $h(x)=1$ for every vertex $x$ of $H$ and so $\sigma_{h}(x)=\operatorname{deg} x$ for every vertex $x$ of $H$. Since $\{\operatorname{deg} x: x \in V(H)\}=S_{h}=[n-1]$, it follows that $H=G_{n}$ is the connected antiregular graph of order $n$.

Case 2. $h(u)=0$. Therefore, $u$ is the only vertex of $H$ whose $h$-value is 0 . This implies that $1 \leq \sigma_{h}(x)=\operatorname{deg}_{H} x-1 \leq n-2$ for every vertex $x$ of $V(H)-\{u\}$. Thus, $u$ is the only vertex of $H$ whose $\sigma_{h}$-value is $n-1$ and so

$$
\left\{\sigma_{h}(x): x \in V(H)-\{u\}\right\}=\left\{\operatorname{deg}_{H} x-1: x \in V(H)-\{u\}\right\}=[n-2] .
$$

Then $H^{\prime}=H-u$ is a connected subgraph of $H$ and $\operatorname{deg}_{H^{\prime}} x=\operatorname{deg}_{H} x-1$ for each vertex $x$ of $H^{\prime}$. Since the order of $H^{\prime}$ is $n-1$ and $\left\{\operatorname{deg}_{H^{\prime}} x: x \in V\left(H^{\prime}\right)\right\}=[n-2]$, it follows that $H^{\prime}$ is the connected antiregular graph $G_{n-1}$ of order $n-1$. Therefore, $H=H^{\prime} \vee K_{1}=G_{n-1} \vee K_{1}$.

Therefore, the two graphs of order $n \geq 3$ with an antiregular total dominating function are $G_{n}$ and $G_{n-1} \vee K_{1}$.

If $n=4$, then $G_{4}$ and $G_{3} \vee K_{1}=P_{3} \vee K_{1}$ (which is also shown in Figure 2) are the only connected graphs of order 4 that have an antiregular total dominating function. If $n=5$, then $G_{5}$ and $G_{4} \vee K_{1}$ (which is also shown in Figure 2) are the only connected graphs of order 5 that have an antiregular total dominating function. These four graphs are shown in Figure 3 together with an antiregular total dominating function for each of these four graphs.

By Theorem 2.1, for each integer $n \geq 3$, there is a graph $G$ of order $n$ having a total dominating function $h$ such that $\left\{\sigma_{h}(v): v \in V(G)\right\}=[n-1]$. This gives rise to the following question.

For a given nonempty set $S$ of positive integers, does there exist a graph $G$ with a total dominating function $h$ such that $\left\{\sigma_{h}(v): v \in V(G)\right\}=S$ ? 

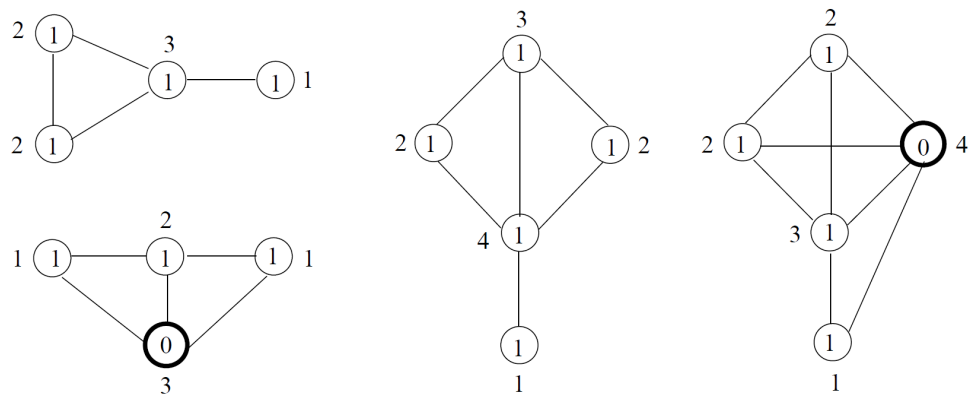

Figure 3: The four graphs of order 4 or 5 having an antiregular total dominating function.

It turns out that this question has an affirmative answer. In 1977, Kapoor, Polimeni, and Wall [14] proved that every set of positive integers is the degree set of some graph.

Theorem 2.2. For every set $S=\left\{a_{1}, a_{2}, \ldots, a_{k}\right\}$ of positive integers with $a_{1}<a_{2}<\cdots<a_{k}$, there exists a graph $G$ whose degree set is $S$. Furthermore, the minimum order of such a graph $G$ is $a_{k}+1$.

As an immediate consequence of Theorem 2.2, every nonempty set of positive integers is realizable as the set of $\sigma_{h}$-values of a total dominating function $h$ of some graph.

Corollary 2.1. For every set $S=\left\{a_{1}, a_{2}, \ldots, a_{k}\right\}$ of positive integers with $a_{1}<a_{2}<\cdots<a_{k}$, there exists a graph $G$ of order $a_{k}+1$ having a total dominating function $h$ such that

$$
\left\{\sigma_{h}(v): v \in V(G)\right\}=S .
$$

Proof. Let $S=\left\{a_{1}, a_{2}, \ldots, a_{k}\right\}$ be any set of positive integers with $a_{1}<a_{2}<\cdots<a_{k}$. By Theorem 2.2, there exists a graph $G$ of order $a_{k}+1$ whose degree set is $S$. Let $h: V(G) \rightarrow\{0,1\}$ be defined by $h(v)=1$ for every vertex $v$ of $G$. Since $\sigma_{h}(v)=\operatorname{deg}(v) \geq 1$ for every $v \in V(G)$, it follows that $h$ is a total dominating function of $G$ and $\left\{\sigma_{h}(v): v \in V(G)\right\}=S$.

\section{Regular total dominating functions}

If $h$ is a total dominating function of a graph $G$ and $\sigma_{h}(v)$ is the same constant $k$ for every vertex $v$ of $G$, then $h$ is called a regular total dominating function (or $k$-regular total dominating function) of $G$. Not every graph without isolated vertices has a $k$-regular total dominating function for some $k$. For example, the graph $G$ of Figure 4 has no $k$-regular total dominating function for any positive integer $k$. To see this, assume, to the contrary, that $G$ has a $k$-regular total dominating function $h: V(G) \rightarrow\{0,1\}$ for some positive integer $k$. Since $u$ is only dominated by $v$ and $x$ is only dominated by $w$, it follows that $h(v)=h(w)=1$. Since $\sigma_{h}(u)=\sigma_{h}(x)=1$, it follows that $k=1$. On the other hand, $\sigma_{h}(y)=h(v)+h(w)=2$, which is impossible.

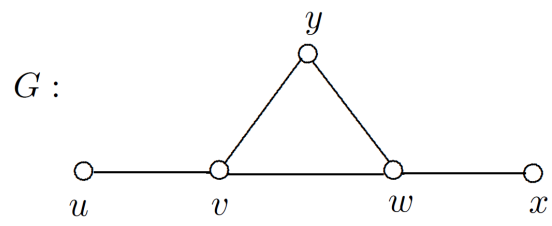

Figure 4: A graph $G$ with no regular total dominating function.

The primary question here is that of determining which graphs (especially those belonging to well-known classes of graphs) have a $k$-regular total dominating function for some $k \in \mathbb{N}$ and which do not. We first answer this question for complete multipartite graphs.

Proposition 3.1. For an integer $\ell \geq 2$, every complete $\ell$-partite graph has a $k$-regular total dominating function for each integer $k$ with $1 \leq k \leq \ell-1$. In particular, every complete graph $K_{n}$ of order $n \geq 2$ has a $k$-regular total dominating function for each integer $k$ with $1 \leq k \leq n-1$.

Proof. Let $G$ be a complete $\ell$-partite graph where $\ell \geq 2$. For each integer $k$ with $1 \leq k \leq \ell$, let $H_{k}$ be the complete subgraph of order $k$ in $G$. Then a $(k-1)$-regular total dominating function $h_{k}: V(G) \rightarrow\{0,1\}$ of $G$ can be defined by $h_{k}(v)=1$ if $v \in V\left(H_{k}\right)$ and $h_{k}(v)=0$ if $v \in V(G)-V\left(H_{k}\right)$. 
We begin by presenting some preliminary results dealing with regular total dominating functions of graphs.

Observation 3.1. Let $G$ be a nontrivial connected graph.

(1) If $G$ is an $r$-regular graph for some positive integer $r$, then the function h defined by $h(x)=1$ for each vertex $x$ of $G$ is an r-regular total dominating function of $G$.

(2) If $G$ has a k-regular total dominating function, then $1 \leq k \leq \delta(G)$.

(3) Let $H$ be a graph with a regular total dominating function $h$ and let $v$ be a vertex of $H$ with $h(v)=0$. If $G$ is the graph obtained by replacing $v$ by an arbitrary graph $F$ where each vertex of $F$ is joined to the neighbors of $v$, then $h$ can be extended to a regular total dominating function of $G$ by defining $h(x)=0$ for each $x \in V(F)$.

Proposition 3.2. For a positive integer $k$, a graph $G$ has a $k$-regular total dominating function if and only if either $G$ is $k$-regular or $G$ consists of two induced vertex-disjoint subgraphs $F$ and $H$ such that $F$ is $k$-regular and each vertex of $H$ is adjacent to exactly $k$ vertices in $F$.

Proof. First, suppose that $G$ has a $k$-regular total dominating function $h: V(G) \rightarrow\{0,1\}$ for some positive integer $k$. To simplify the notation, we let

$$
\mathcal{I}_{h}=\{v \in V(G): h(v)=1\} \text { and } \overline{\mathcal{I}_{h}}=V(G)-\mathcal{I}_{h}=\{v \in V(G): h(v)=0\} .
$$

Next, let $F=G\left[\mathcal{I}_{h}\right]$ and $H=G\left[\overline{\mathcal{I}_{h}}\right]$ (if $\overline{\mathcal{I}_{h}} \neq \emptyset$ ). Since $\sigma_{h}(v)=k$ for each $v \in V(G)$, it follows that every vertex in $F$ is adjacent to exactly $k$ vertices in $F$ and so $F$ is $k$-regular and every vertex in $H$ is adjacent to exactly $k$ vertices in $F$.

Next, we verify the converse. Since the statement is true if $G$ is $k$-regular, we may assume that $G$ is constructed from two induced vertex-disjoint subgraphs $F$ and $H$ such that $F$ is $k$-regular and each vertex of $H$ is adjacent to exactly $k$ vertices in $F$. Then a $k$-regular total dominating function of $G$ can be defined by assigning 0 to each vertex of $H$ and assigning 1 to each vertex of $F$.

* If $k=1$ in Proposition 3.2, then a nonempty graph $G$ has a 1-regular total dominating function if and only if $F$ is 1-regular (or $E(F)$ is a matching in $G$ ) and each vertex in $H$ is adjacent to exactly one vertex of $F$.

$\star$ If $k=2$ in Proposition 3.2, then $G$ has a 2-regular dominating function if and only if $F$ is 2-regular (or the subgraph induced by $E(F)$ is a union of cycles) and each vertex in $H$ is adjacent to exactly two vertices of $F$.

The following is a consequence of Observation 1.1.

Proposition 3.3. Let $G$ be a nontrivial connected graph of order $n$, let $h: V(G) \rightarrow\{0,1\}$ be a total dominating function of $G$, and suppose that $\left|\mathcal{I}_{h}\right|=s \geq 2$.

(1) If $G$ is an r-regular graph for some integer $r \geq 2$, then

$$
\sum_{v \in V(G)} \sigma_{h}(v)=r s .
$$

(2) If $h$ is a k-regular total dominating function for some integer $k \geq 1$, then

$$
\sum_{v \in V(G)} \sigma_{h}(v)=n k .
$$

Proof. By Proposition 1.1, it follows that if $G$ is an $r$-regular graph for some integer $r \geq 2$, then

$$
\sum_{v \in V(G)} \sigma_{h}(v)=\sum_{x \in \mathcal{I}_{h}} \operatorname{deg} x=\sum_{x \in \mathcal{I}_{h}} r=r s
$$

and so (1) holds. Also, by Proposition 1.1, if $h$ is a $k$-regular total dominating function of a graph $G$ for some integer $k \geq 1$, then

and so (2) holds.

$$
\sum_{v \in V(G)} \sigma_{h}(v)=\sum_{v \in V(G)} k=n k
$$

For two vertices $u$ and $v$ in a connected graph $G$, the distance $d(u, v)$ between $u$ and $v$ is the length of a shortest $u-v$ path in $G$. For a vertex $v$ in the graph $G$, the eccentricity $e(v)$ of $v$ is the distance between $v$ and a vertex farthest from $v$ in $G$. The minimum eccentricity among the vertices of $G$ is its radius and the maximum eccentricity is its diameter, which are denoted by $\operatorname{rad}(G)$ and $\operatorname{diam}(G)$, respectively. A vertex $v$ in $G$ is a central vertex if $e(v)=\operatorname{rad}(G)$; while $v$ is a peripheral vertex of $G$ if $e(v)=\operatorname{diam}(G)$. 
Observation 3.2. Let $G$ be a connected graph having a 1-regular total dominating function $h$ and let $u$ be an end-vertex of $G$.

$\star$ If $v$ is a vertex of $G$ that is adjacent to $u$, then $h(v)=1$.

$\star$ If $w$ is a vertex of $G$ such that $d(u, w)=3$, then $h(w)=0$.

Proposition 3.4. If a connected graph $G$ contains two end-vertices $x$ and $y$ such that $d(x, y)=4$, then $G$ does not have any 1-regular total dominating function.

Proof. Let $(x, u, v, w, y)$ be an $x-y$ path in $G$. By Observation 3.2, any 1-regular total dominating function $h$ must assign 1 to $u$ and $w$. However then, $\sigma_{h}(v) \geq 2$, which is impossible.

\section{Regular total dominating functions in trees}

By Observation 3.1, if a connected graph $G$ has a $k$-regular total dominating function, then $1 \leq k \leq \delta(G)$. Hence, if $T$ is a nontrivial tree, then the only possible regular total dominating function for $T$ is a 1-regular total dominating function. All trees of diameter 3 or less have a 1-regular total dominating function.

Proposition 4.1. Every star and double star has a 1-regular total dominating function.

Only certain paths have a 1-regular dominating function, however.

Proposition 4.2. A path $P_{n}$ of order $n \geq 2$ has a 1-regular total dominating function if and only if $n \not \equiv 1(\bmod 4)$.

Proof. Let $P_{n}=\left(v_{1}, v_{2}, \ldots, v_{n}\right)$. First, suppose that $n \not \equiv 1(\bmod 4)$. For $n \equiv 0(\bmod 4)$, define $h: V\left(P_{n}\right) \rightarrow\{0,1\}$ by

$$
h\left(v_{i}\right)= \begin{cases}0 & \text { if } i \equiv 0,1(\bmod 4) \\ 1 & \text { if } i \equiv 2,3(\bmod 4) .\end{cases}
$$

For $n \equiv 2,3(\bmod 4)$, define $h: V\left(P_{n}\right) \rightarrow\{0,1\}$ by

$$
h\left(v_{i}\right)= \begin{cases}0 & \text { if } i \equiv 0,3(\bmod 4) \\ 1 & \text { if } i \equiv 1,2(\bmod 4) .\end{cases}
$$

In each case, $h$ is a 1-regular total dominating function of $P_{n}$.

For the converse, assume, to the contrary, that there is an integer $n \geq 5$ with $n \equiv 1(\bmod 4) \operatorname{such}$ that $P_{n}$ has a 1-regular total dominating function $h: V\left(P_{n}\right) \rightarrow\{0,1\}$. Since $n$ is odd and

$$
\sum_{v \in V(G)} \sigma_{h}(v)=\sum_{x \in \mathcal{I}_{h}} \operatorname{deg} x=n
$$

by Propositions 1.1 and 3.3, it follows that exactly one of $v_{1}$ and $v_{n}$ belongs to $\mathcal{I}_{h}$, say $v_{1} \in \mathcal{I}_{h}$ and $v_{n} \notin \mathcal{I}_{h}$. Since $v_{1} \in \mathcal{I}_{h}$, this forces

$$
h\left(v_{i}\right)= \begin{cases}1 & \text { if } i \equiv 1,2(\bmod 4) \\ 0 & \text { if } i \equiv 3,0(\bmod 4)\end{cases}
$$

and so $v_{n} \in \mathcal{I}_{h}$, which is a contradiction.

The following are consequences of Propositions 3.4 and 4.2, respectively.

Corollary 4.1. If a tree $T$ contains two end-vertices $x$ and $y$ such that $d(x, y)=4$, then $T$ does not have any 1-regular total dominating function. In particular, if $\operatorname{diam}(T)=4$, then $T$ does not have any 1-regular total dominating function.

Corollary 4.2. If $G$ is a path with $\operatorname{diam}(G) \equiv 0(\bmod 4)$, then $G$ does not have a 1-regular total dominating function.

Corollary 4.2 gives rise to the following question.

If $T$ is a tree with diameter $d \geq 1$ where $d \not \equiv 0(\bmod 4)$, does $T$ have a 1-regular total dominating function?

The answer to this question depends on the structure of the tree in question, as we will soon see. However, every positive integer $d$ distinct from 4 can be realized as the diameter of a tree $T$ having a 1-regular total dominating function.

Proposition 4.3. For every positive integer $d$ distinct from 4, there exists a tree of diameter $d$ having a 1-regular total dominating function. 


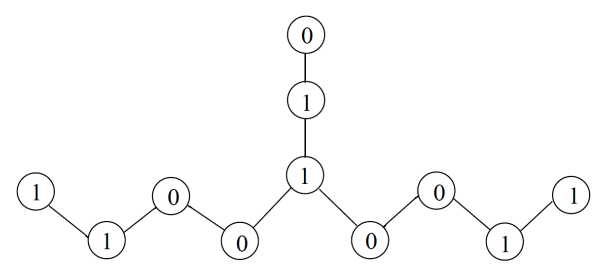

Figure 5: A tree of diameter 8 having a 1-regular total dominating function.

Proof. If $d$ is a positive integer such that $d \not \equiv 0(\bmod 4)$, then the path $P_{d+1}$ of diameter $d$ has a 1-regular total dominating function by Proposition 4.2. Thus, we may assume that $d \equiv 0(\bmod 4)$ and $d \geq 8$. Then $d=4 q$ for some integer $q \geq 2$. We show that there is a tree $T_{q}$ of diameter $4 q$ that has a 1-regular total dominating function $h_{q}$. Figure 5 shows the tree $T_{2}$ of diameter 8 having a 1-regular total dominating function $h_{2}$. Thus, the statement is true for $q=2$.

For an integer $q \geq 3$, let $T_{q}$ be the tree obtained from the tree $T_{2}$ of Figure 5 and the path $P_{4 q-8}=\left(v_{1}, v_{2}, \ldots, v_{4 q-8}\right)$ of order $4 q-8$ by adding the edge $v v_{1}$ where $v$ is an end-vertex with $h_{2}(v)=1$. Then $\operatorname{diam}\left(T_{q}\right)=8+(4 q-8)=4 q$. The function $h_{q}: V\left(T_{q}\right) \rightarrow\{0,1\}$ of $T_{q}$ defined by $h_{q}(x)=h_{2}(x)$ if $x \in V\left(T_{2}\right)$ and

$$
h_{q}\left(v_{i}\right)= \begin{cases}0 & \text { if } i \equiv 1,2(\bmod 4) \\ 1 & \text { if } i \equiv 0,3(\bmod 4)\end{cases}
$$

is a 1-regular total dominating function of $T_{q}$. This is illustrated in Figure 6 for the tree $T_{3}$ of diameter 12 together with 1-regular total dominating function $h_{3}$.

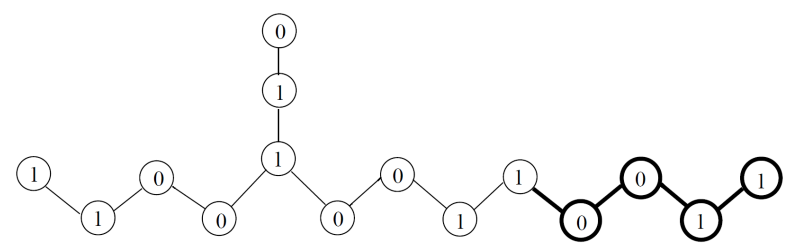

Figure 6: A tree of diameter 12 having a 1-regular total dominating function.

In fact, if $T$ is a tree with a 1-regular total dominating function $h$ and $v$ is a vertex of $T$ with $h(v)=1$, then the tree $T^{\prime}$ obtained from $T$ by adding a new vertex $u$ and joining $u$ to the vertex $v$ also has a 1-regular total dominating function. For example, a 1-regular total dominating function $h^{\prime}$ of $T^{\prime}$ can be defined by $h^{\prime}(x)=h(x)$ if $x \in V(T)$ and $h^{\prime}(u)=0$. Consequently, for every positive integer $d \geq 2$ distinct from 4 , there exist infinitely many trees of diameter $d$ having a 1-regular total dominating function.

Next, we characterize those trees having a 1-regular total dominating function. To do this, we first introduce additional terminology. A tree $T$ is called totally 1-sequential if the tree $T$ with a 1-regular total dominating function $h: V(T) \rightarrow\{0,1\}$ can be constructed by means of the following algorithm.

Algorithm 4.1. Constructing a totally 1-sequential tree T.

1. We begin with $T_{0}=K_{2}$ whose two vertices are labeled 1 .

2. Once a tree $T_{j}, j \geq 0$, has been constructed with a 1-regular total dominating function $h$, a tree $T_{j+1}$ with an extended 1-regular total dominating function $h$ is constructed by performing one of the following steps:

(2.1) A vertex $v$ is added to $T_{j}$ where $v$ is joined to a vertex labeled 1 in $T_{j}$ and $h(v)$ is defined to be 0 .

(2.2) A path $(x, y, z)$ of order 3 is added to $T_{j}$ where $x$ is joined to a vertex labeled 0 in $T_{j}, h(x)$ is defined to be 0 , and $h(y)$ and $h(z)$ are defined to be 1.

3. Either repeat Step 2 or stop, resulting in a tree $T=T_{k}$ for some nonnegative integer $k$.

Once Algorithm 4.1 stops, a sequence of labeled trees $T_{0}, T_{1}, T_{2}, \ldots, T_{k}$ is constructed, resulting in $T_{k} \cong T$. Such a tree $T$ is therefore totally 1-sequential. Our interest in totally 1-sequential trees is due to the following result.

Theorem 4.1. A tree $T$ has a 1-regular total dominating function if and only if $T$ is totally 1-sequential. 
Proof. First, if $T$ is a totally 1-sequential tree, then from the manner in which $T$ is constructed, we see that the defined function $h$ is a 1-regular total dominating function of $T$. For the converse, assume, to the contrary, that there are trees possessing a 1-regular total dominating function that are not totally 1-sequential. Among all such trees, let $T$ be one of minimum order $n$. Since it is clear that all trees of order 6 or less that possess a 1-regular total dominating function are totally 1-sequential, it follows that $n \geq 7$. Let $h$ be a 1-regular total dominating function of $T$.

First, suppose that $T$ contains an end-vertex $u$ such that $h(u)=0$. Suppose that $u$ is adjacent to the vertex $v$ in $T$. Necessarily, $h(v)=1$. The restriction of the 1-regular dominating function $h$ to the tree $T-u$ of order $n-1$ is a 1-regular total dominating function of $T-u$. Thus, $T-u$ is totally 1-sequential. Adding the vertex $u$ to $T-u$, joining $u$ to $v$, and defining $h(u)=0$ shows that $T$ is totally 1 -sequential, producing a contradiction. Hence, we may assume that $h(w)=1$ for every end-vertex $w$ of $T$.

Let $z$ be a peripheral vertex of $T$. Since $z$ is an end-vertex of $T$, it follows that $h(z)=1$. Let $y$ be the neighbor of $z$ in $T$. Thus, $h(y)=1$. If $y$ is adjacent to an end-vertex $v$ distinct from $z$, then $h(v)=0$, contrary to our assumption. Thus, $z$ is the only end-vertex adjacent to $y$. Since $z$ is a peripheral vertex of $T$, it follows that $\operatorname{deg}_{T} y=2$. Let $(x, y, z)$ be a path in $T$. Then $h(x)=0$. Since $\sigma_{h}(x)=1$ and $h(y)=1$, it follows that every neighbor of $x$ distinct from $y$ must be labeled 0 by $h$. We claim that $\operatorname{deg}_{T} x=2$, for suppose that $\operatorname{deg}_{T} x \geq 3$. Let $u$ and $w$ be two neighbors of $x$ distinct from $y$. Thus, $h(u)=h(w)=0$. Since $\sigma_{h}(u)=\sigma_{h}(w)=1$, it follows that each of $u$ and $w$ has a neighbor $u^{\prime}$ and $w^{\prime}$, respectively, such that $h\left(u^{\prime}\right)=h\left(w^{\prime}\right)=1$. Since $\sigma_{h}\left(u^{\prime}\right)=\sigma_{h}\left(w^{\prime}\right)=1$, it follows that each of $u^{\prime}$ and $w^{\prime}$ has a neighbor $u^{\prime \prime}$ and $w^{\prime \prime}$, respectively, such that $h\left(u^{\prime \prime}\right)=h\left(w^{\prime \prime}\right)=1$. This, however, contradicts the fact that $z$ is a peripheral vertex of $T$. Therefore, as claimed, $\operatorname{deg}_{T} x=2$. Let $u$ be the neighbor of $x$ distinct from $y$. We saw that $h(u)=0$. Then $T^{\prime}=T-\{x, y, z\}$ is a tree of order $n-3$ and the restriction of the 1-regular total dominating function $h$ of $T$ to $T^{\prime}$ is a 1-regular total dominating function of $T^{\prime}$. Hence, $T^{\prime}$ is totally 1-sequential. Adding the path $(x, y, z)$ to $T^{\prime}$, joining $x$ to $u$, and defining $h(x)=0$ and $h(y)=h(z)=1$ shows that $T$ is totally 1 -sequential, producing a contradiction.

\section{Regular total dominating functions in regular graphs}

Let $G$ be a nontrivial connected graph and let $h: V(G) \rightarrow\{0,1\}$ be a total dominating function of $G$. The complementary function $\bar{h}: V(G) \rightarrow\{0,1\}$ is defined by

$$
\bar{h}(v)=1-h(v) \text { for every vertex } v \text { of } G .
$$

Proposition 5.1. Let $G$ be a nontrivial connected graph. If $h: V(G) \rightarrow\{0,1\}$ is a total dominating function of $G$, then $\sigma_{h}(v)+\sigma_{\bar{h}}(v)=\operatorname{deg} v$ for each vertex $v$ of $G$.

Proof. Let $v \in V(G)$ where $h(v)=i \in\{0,1\}$ and $\sigma_{h}(v)=k$. Then $\bar{h}(v)=1-i$. Since $\sigma_{h}(v)=k$, it follows that $v$ is adjacent to $k$ vertices labeled 1 by $h$ and so $v$ is adjacent to $\operatorname{deg} v-k$ vertices labeled 0 by $h$. Thus, $v$ is adjacent to $\operatorname{deg} v-k$ vertices labeled 1 by $\bar{h}$. Hence, $\sigma_{\bar{h}}(v)=\operatorname{deg} v-k$. Consequently, $\sigma_{h}(v)+\sigma_{\bar{h}}(v)=\operatorname{deg} v$.

The following two corollaries are consequences of Proposition 5.1.

Corollary 5.1. Let $G$ be a nontrivial connected graph. If $h: V(G) \rightarrow\{0,1\}$ is a total dominating function of $G$ such that $\sigma_{h}(v) \leq \operatorname{deg} v-1$ for each vertex $v$ of $G$, then its complementary function $\bar{h}$ is also a total dominating function of $G$.

Corollary 5.2. Let $G$ be a nontrivial connected graph. Suppose that $h$ is a total dominating function of $G$ such that $\bar{h}$ is also a total dominating function of $G$. Then $h$ and $\bar{h}$ are both regular if and only if $G$ is regular. Furthermore, if $G$ is an r-regular graph and $h$ is a k-regular total dominating function of $G$ where $1 \leq k \leq r$, then $\bar{h}$ is an $(r-k)$-regular total dominating function of $G$.

By Corollary 5.2, we have the following corollary.

Corollary 5.3. For a nontrivial connected r-regular graph $G$ for some integer $r \geq 2$, a function $h: V(G) \rightarrow\{0,1\}$ is a 1-regular total dominating function of $G$ if and only if $\bar{h}: V(G) \rightarrow\{0,1\}$ is an $(r-1)$-regular total dominating function of $G$.

For each integer $n \geq 3$, every cycle $C_{n}$ of order $n$ is 2-regular and so has a 2-regular total dominating function. Next, we determine those $n$-cycles having a 1-regular total dominating function.

Proposition 5.2. For an integer $n \geq 3$, the $n$-cycle $C_{n}$ has a 1-regular total dominating function if and only if $n \equiv 0(\bmod 4)$. 
Proof. First, suppose that $C_{n}$ has a 1-regular total dominating function $h$. Let $\mathcal{I}_{h}=\{v \in V(G): h(v)=1\}$. Since the edge set of $G\left[\mathcal{I}_{h}\right]$ is a matching, it follows that $\left|\mathcal{I}_{h}\right|=s$ is even. Since $C_{n}$ is a 2-regular graph of order $n$ and $s$ is even, it follows by Observation 3.3 that

$$
n=\sum_{v \in V(G)} \sigma_{h}(v)=2 s \equiv 0(\bmod 4) .
$$

For the converse, suppose that $n \equiv 0(\bmod 4)$ and let $C_{n}=\left(v_{1}, v_{2}, \ldots, v_{n}, v_{1}\right)$. A 1-regular total dominating function $f: V\left(C_{n}\right) \rightarrow\{0,1\}$ of $G$ can be defined by $f\left(v_{i}\right)=1$ if $i \equiv 0,1(\bmod 4)$ and $f\left(v_{i}\right)=0$ if $i \equiv 2,3(\bmod 4)$.

We now turn our attention from connected 2-regular graphs (cycles) to connected 3-regular (cubic) graphs. While every connected cubic graph has a 3-regular total dominating function, there is no guarantee that a cubic graph has a $k$-regular total dominating function for $k \in\{1,2\}$. We first present a necessary condition on the order of a cubic graph to possess a $k$-regular total dominating function for $k \in\{1,2\}$.

Lemma 5.1. If $G$ is a connected cubic graph of order $n$ having a k-regular total dominating function where $k \in\{1,2\}$, then $n \equiv 0(\bmod 6)$.

Proof. Suppose that $G$ has a $k$-regular total dominating function $h$, where $k \in\{1,2\}$. Let $|\{v \in V(G): h(v)=1\}|=s$. Since $G$ is a 3 -regular graph of order $n$, it follows by Observation 3.3 that

$$
n k=\sum_{v \in V(G)} \sigma_{h}(v)=3 s .
$$

If $k=1$, then $n=3 s$, while if $k=2$, then $2 n=3 s$. In either case, $n \equiv 0(\bmod 3)$. Since $n$ is even, it follows that $n \equiv 0$ $(\bmod 6)$.

As a consequence of Lemma 5.1, no cubic graph of order 10 has a 1-regular total dominating function or a 2-regular total dominating function. In particular, the Petersen graph has neither a 1-regular total dominating function nor a 2-regular total dominating function.

Corollary 5.4. The Petersen graph has a k-regular total dominating function if and only if $k=3$.

The Cartesian product $G \square H$ of two graphs $G$ and $H$ has vertex set $V(G \square H)=V(G) \times V(H)$ and two distinct vertices $(u, v)$ and $(x, y)$ of $G \square H$ are adjacent if either (1) $u=x$ and $v y \in E(H)$ or (2) $v=y$ and $u x \in E(G)$. One of the best known classes of cubic graphs is that of the prisms $C_{n} \square K_{2}$.

Proposition 5.3. Let $n \geq 3$ and $k \in\{1,2\}$. Then $C_{n} \square K_{2}$ has a k-regular total dominating function if and only if $n \equiv 0$ $(\bmod 3)$.

Proof. Let $G=C_{n} \square K_{2}$, where $n \geq 3$. First, suppose that $G$ has a $k$-regular total dominating function $h$, where $k \in\{1,2\}$. Since $G$ is a 3-regular graph of order $2 n$, it follows by Lemma 5.1 that $2 n \equiv 0(\bmod 3)$ and so $n \equiv 0(\bmod 3)$.

For the converse, suppose that $n \equiv 0(\bmod 3)$. Let $G$ be constructed from two copies $\left(u_{1}, u_{2}, \ldots, u_{n}, u_{1}\right)$ and $\left(v_{1}, v_{2}, \ldots\right.$, $\left.v_{n}, v_{1}\right)$ of the $n$-cycle by adding the edges $u_{i} v_{i+1}$ for $1 \leq i \leq n$. The function $h_{1}: V(G) \rightarrow\{0,1\}$ of $G$ defined by

$$
h_{1}(w)= \begin{cases}1 & \text { if } w=u_{i} \text { or } w=v_{i} \text { where } i \equiv 1(\bmod 3) \\ 0 & \text { otherwise }\end{cases}
$$

is a 1-regular total dominating function of $G$ and $\bar{h}_{1}$ is a 2-regular total dominating function of $G$ by Corollary 5.2.

A class of graphs related to the prisms $C_{n} \square K_{2}$ are the graphs $P_{n} \square K_{2}$, sometimes referred to as ladders.

Proposition 5.4. For each positive integer $n$, the ladder graph $P_{n} \square K_{2}$ has a 1-regular total dominating function.

Proof. Let $G=P_{n} \square K_{2}$ be constructed from the two copies $\left(u_{1}, u_{2}, \ldots, u_{n}\right)$ and $\left(v_{1}, v_{2}, \ldots, v_{n}\right)$ of the path $P_{n}$ of order $n$ by adding the edges $u_{i} v_{i}$ for $1 \leq i \leq n$. If $n \equiv 1(\bmod 3)$, then the function $h: V(G) \rightarrow\{0,1\}$ defined by

$$
h(w)= \begin{cases}1 & \text { if } w=u_{i} \text { or } w=v_{i} \text { where } i \equiv 1(\bmod 3) \\ 0 & \text { otherwise }\end{cases}
$$

is a 1-regular total dominating function of $G$. If $n \not \equiv 1(\bmod 3)$, then the function $h: V(G) \rightarrow\{0,1\}$ defined by

$$
h(w)= \begin{cases}1 & \text { if } w=u_{i} \text { or } w=v_{i} \text { where } i \equiv 2(\bmod 3) \\ 0 & \text { otherwise }\end{cases}
$$

is a 1-regular total dominating function of $G$. 
Proposition 5.5. For a positive integer $n$, the ladder graph $P_{n} \square K_{2}$ has a 2-regular total dominating function if and only if $n \equiv 2(\bmod 3)$.

Proof. Let $G=P_{n} \square K_{2}$ be constructed from the two copies $\left(u_{1}, u_{2}, \ldots, u_{n}\right)$ and $\left(v_{1}, v_{2}, \ldots, v_{n}\right)$ of the path $P_{n}$ of order $n$ by adding the edges $u_{i} v_{i}$ for $1 \leq i \leq n$. If $n \equiv 2(\bmod 3)$, then the function $h: V(G) \rightarrow\{0,1\}$ defined by

$$
h(w)= \begin{cases}0 & \text { if } w=u_{i} \text { or } w=v_{i} \text { where } i \equiv 0(\bmod 3) \\ 1 & \text { otherwise }\end{cases}
$$

is a 2-regular total dominating function of $G$.

For the converse, assume, to the contrary, that there is a positive integer $n$ such that $n \not \equiv 2(\bmod 3)$ and $G=P_{n} \square K_{2}$ has a 2-regular total dominating function $h$. Because $P_{1} \square K_{2}=K_{2}$ does not have a 2-regular total dominating function, it follows that $n \geq 3$. Since $\operatorname{deg} u_{1}=\operatorname{deg} v_{1}=2$ and $\sigma_{h}\left(u_{1}\right)=\sigma_{h}\left(v_{1}\right)=2$, it follows that $h(x)=1$ for each $x \in N\left(u_{1}\right) \cup$ $N\left(v_{1}\right)=\left\{u_{1}, u_{2}, v_{1}, v_{2}\right\}$. Because $\sigma_{h}\left(u_{2}\right)=\sigma_{h}\left(v_{2}\right)=2$, we have $h\left(u_{3}\right)=h\left(v_{3}\right)=0$. If $n=3$, then $\sigma_{h}\left(u_{3}\right)=\sigma_{h}\left(v_{3}\right)=1$, a contradiction. Thus, $n \geq 4$ and $h\left(u_{4}\right)=h\left(v_{4}\right)=1$. If $n=4$, then $\sigma_{h}\left(u_{4}\right)=\sigma_{h}\left(v_{4}\right)=1$, a contradiction. Thus, $n \geq 6$ and $h\left(u_{5}\right)=h\left(v_{5}\right)=h\left(u_{6}\right)=h\left(v_{6}\right)=1$. Continuing in this fashion, we see that $h\left(u_{i}\right)=h\left(v_{i}\right)=0$ if $i \equiv 0(\bmod 3)$ and $h\left(u_{i}\right)=h\left(v_{i}\right)=1$ if $i \not \equiv 0(\bmod 3)$. If $n \equiv 0(\bmod 3)$, then $N\left(v_{n}\right)=\left\{u_{n}, v_{n-1}\right\}$ and $h\left(u_{n}\right)=0$, which implies that $\sigma_{h}\left(v_{n}\right)=1$; while if $n \equiv 1(\bmod 3)$, then $N\left(v_{n}\right)=\left\{u_{n}, v_{n-1}\right\}$ and $h\left(v_{n-1}\right)=0$, which implies that $\sigma_{h}\left(v_{n}\right)=1$. A contradiction is produced in either case. Therefore, if $n \not \equiv 2(\bmod 3)$, then $P_{n} \square K_{2}$ does not have a 2-regular total dominating function.

We conclude with the following question.

Problem 5.1. Under what conditions do both the graphs $G$ and $G \square K_{2}$ have a k-regular total dominating function for some positive integer $k$ ?

\section{Acknowledgments}

We are grateful to Professor Gary Chartrand for suggesting the concept of total dominating functions to us and kindly providing useful information on this topic. Furthermore, we thank the anonymous referees whose valuable suggestions resulted in an improved paper.

\section{References}

[1] A. Ali, A survey of antiregular graphs, Contrib. Math. 1 (2020) 67-79.

[2] A. Ali, G. Chartrand, P. Zhang, Irregularity in Graphs, Springer, New York, 2021.

[3] C. Berge, Sur le couplage maximum d'un graphe, C. R. Acad. Sci. Paris 247 (1958) 258-259.

[4] G. Chartrand, T. W. Haynes, M. A. Henning, P. Zhang, From Domination to Coloring: Stephen Hedetniemi's Graph Theory and Beyond, Springer, New York, 2019.

[5] G. Chartrand, P. Zhang, Chromatic Graph Theory, Second Edition, Chapman \& Hall/CRC Press, Boca Raton, 2020

[6] E. J. Cockayne, R. M. Dawes, S. T. Hedetniemi, Total domination in graphs, Networks 10 (1977) 211-219.

[7] E. J. Cockayne, S. T. Hedetniemi, Towards a theory of domination in graphs, Networks 7 (1977) 247-261.

[8] J. Hallas, M. Talanda-Fisher, P. Zhang, Dominating functions in graphs-regularity versus irregularity, Int. J. Comput. Math. Comput. Syst. Theory 5 (2020) 98-111.

[9] J. Hallas, M. Talanda-Fisher, P. Zhang, Proper dominating functions of graphs, Contrib. Math. 2 (2020) 1-10.

[10] J. Hallas, M. Talanda-Fisher, P. Zhang, Regular dominating functions in regular graphs, Bull. Inst. Combin. Appl. 90 (2020) $139-157$.

[11] L. Kang, E. Shan, Dominating functions with integer values in graphs-a survey, J. Shanghai Univ. 11 (2007) $437-448$.

[12] T. W. Haynes, S. T. Hedetniemi, P. J. Slater, Fundamentals of Domination in Graphs, Marcel Dekker, New York, 1998.

[13] T. W. Haynes, S. T. Hedetniemi, P. J. Slater (Eds.), Domination in Graphs: Advanced Topics, Marcel Dekker, New York, 1998.

[14] S. F. Kapoor, A. D. Polimeni, C. E. Wall, Degree sets for graphs. Fund. Math. 95 (1977) 189-194.

[15] O. Ore, Theory of Graphs, Amer. Math. Soc. Colloq. Pub., Providence, 1962. 